\title{
Video Article \\ Physiological Experimentation with the Crayfish Hindgut: A Student Laboratory Exercise
}

\author{
Ann S. Cooper* ${ }^{*}$, Bonnie Leksrisawat ${ }^{* 1}$, Allison B. Gilberts ${ }^{* 1}$, A. Joffre Mercier* ${ }^{2}$, Robin L. Cooper* \\ ${ }^{1}$ Department of Biology, University of Kentucky \\ ${ }^{2}$ Department of Biological Sciences, Brock University \\ * These authors contributed equally
}

Correspondence to: Robin L. Cooper at RLCOOP1@email.uky.edu

URL: https://www.jove.com/video/2324

DOI: doi:10.3791/2324

Keywords: Neuroscience, Issue 47, invertebrate, crayfish, student laboratory, physiology

Date Published: $1 / 18 / 2011$

Citation: Cooper, A.S., Leksrisawat, B., Gilberts, A.B., Mercier, A.J., Cooper, R.L. Physiological Experimentation with the Crayfish Hindgut: A Student Laboratory Exercise. J. Vis. Exp. (47), e2324, doi:10.3791/2324 (2011).

\section{Abstract}

The purpose of the report is to describe dissection techniques for preparing the crayfish hindgut and to demonstrate how to make physiological recordings with a force transducer to monitor the strength of contraction. In addition, we demonstrate how to visually monitor peristaltic activity, which can be used as a bioassay for various peptides, biogenic amines and neurotransmitters. This preparation is amenable to student laboratories in physiology and for demonstrating pharmacological concepts to students. This preparation has been in use for over 100 years, and it still offers much as a model for investigating the generation and regulation of peristaltic rhythms and for describing the mechanisms underlying their modulation. The pharmacological assays and receptor sub-typing that were started over 50 years ago on the hindgut still contribute to research today. This robust preparation is well suited to training students in physiology and pharmacology.

\section{Video Link}

The video component of this article can be found at https://www.jove.com/video/2324/

\section{Protocol}

\section{Introduction}

The most recent comprehensive overview of the crayfish hindgut was complied in a dissertation by Dr. Barbara E. Musolf (2007, Georgia State University, Atlanta, Georgia, USA), who focused primarily on serotonergic modulation and innervation (Musolf et al., 2009). Crustacean hindguts were first studied over 100 years ago by Alexandrowicz, a pioneer in comparative anatomy (Alexandrowicz, 1909), who described the various aspects of their innervation. Research continued over the years, identifying the types of muscle layers and architecture, addressing the overall function of the hindgut in osmoregulation for the whole animal, and examining modulatory control of hindgut contractility by various compounds (see review by Musolf, 2007). It is interesting to note that the anal portion of the hindgut acts not only to expel feces but also to take up the water from the environment for osmoregulation. This region of the gut can undergo forward or reverse peristalsis depending on the animal's needs.

Alexandrowicz (1909) identified two nerve plexuses innervating the crustacean hindgut, an inner plexus and an outer plexus, which later proved to be rich sources for identifying and characterizing neurotransmitters. In the 1950's Dr. Ernst Florey began a series of pharmacological studies on the crayfish hindgut and demonstrated that contractions are modulated by acetylcholine and its related compounds and also by epinephrine and norepinephrine (Florey, 1954). Florey, the discoverer of an inhibitory substance known as "Factor-I," studied the effects of this substance on various preparations, including the GI system in crayfish (Florey, 1961). Factor-I was later shown to be GABA. Thus, the crayfish hindgut played an important role in the early studies of synaptic inhibition.

Following the discovery of GABA, other putative transmitters were also shown to be associated with the hindgut plexuses and to elicit physiological responses. Such transmitters include dopamine (Elekes et al. 1988; Elofsson et al. 1968), proctolin (RYLPG-OH; Mercier et al. 1997) two orcokinin peptides (NFDEIDRSGFGFN and AFDEIDRSGFGFN; Bungart et al. 1994; Dircksen et al. 2000), orcomyotropin (FDAFTTGFamide; Dircksen et al. 2000) and a myosuppressin peptide (Mercier et al. 1997; most likely pQDLDHVFLRFamide, cf. Stemmler et al. 2007). Each of these substances modulates spontaneous hindgut contractions, and all except GABA appear to be excitatory. The hindgut also responds to glutamate and quisqualate (Jones, 1962; Wrong et al. 2003), but clear evidence for glutamatergic innervation has not been reported. Intracellular messengers that mediate the effects of the various transmitters are largely unexplored, but there is evidence for the involvement of cAMP in dopamine's ability to enhance contractions (Knotz \& Mercier, 1995).

Although the crustacean hindgut contracts spontaneously following denervation, such contractions are typically weak and disorganized (Wales, 1982; Winlow \& Laverack, 1972a). Peristaltic movement requires coordinated motor output from the central nervous system, apparently originating in the last abdominal ganglion (Winlow \& Laverack, 1972a, b, c). In crayfish, the motor output is carried to the hindgut through the $7^{\text {th }}$ 
abdominal root, which contains 75 axons whose cell bodies are localized in the last abdominal ganglion (Kondoh \& Hisada, 1986). At least some of the peptides appear to be supplied to the hindgut plexus from neurons originating in the last abdominal ganglion (Dircksen et al. 2000; Mercier et al. 1991b; Siwicki \& Bishop, 1986), but dopamine is supplied by neurons in more anterior ganglia (Mercier et al. 1991a). Since motor output through the $7^{\text {th }}$ abdominal root is necessary for large, coordinated contractions, it is likely that some or all of the putative transmitters listed above contribute in some way to peristalsis. Their relative contributions, however, are not known. Some insight may be gained by studying their effects on circular and longitudinal muscles separately (Mercier \& Lee, 2002).

In addition to controlling the movement of undigested food, the nerve plexus associated with the crustacean hindgut appears to play an important endocrine function associated with molting. The hindgut of the crab, Carcinus maenas, contains endocrine cells that release crustacean hyperglycemic hormone and its related precursor peptide during ecdysis (Webster et al. 2000), suggesting a role in water uptake and swelling associated with molting. Thus, the crustacean hindgut participates in several important physiological processes.

This report is primarily a teaching tool for advanced high school and undergraduate students in physiology courses that participate in experimentation. The significance as well as the potential mechanism behind how the hindgut contracts and functions can be addressed by the students. Pharmacological agents, neurotransmitters, and neurohormones will be used, and dose-response curves will be constructed, which will engage students in how to acquire and interpret physiological and pharmacological data. A general understanding of receptor function with regard to agonists and antagonists can also be achieved. The students will also learn to present data in graphical form for statistical analysis.

It is very easy to dissect and record contractions from the crayfish hindgut. In the dissected preparation, the gut is easily exposed to exogenous substances. The alteration in peristaltic activity can be monitored visually or with a force transducer, which can also monitor the force of contractions. Because of its simplicity and reliability as a bioassay preparation, the crayfish hindgut is still very useful for investigating many research questions about the mechanisms of peristalsis, modulation of motor output and muscle contraction, and receptor function. The hindgut is also useful for testing insecticides, crustaceancides, and pollutants for specific mechanisms of actions.

\section{Methods}

\section{1) Materials}

- crayfish

- crayfish saline

- dissection instruments (coarse \& fine scissors, coarse \& fine forceps)

- Sylgard-bottomed Petri dish

- steel dissecting pins

- beakers (to hold chemical solutions)

- $\quad$ parafilm (for covering \& mixing solutions)

\section{2) Dissection}

1. Crayfish (Procambarus clarkii) measuring $6-10 \mathrm{~cm}$ in body length should be placed on ice for 5 to 10 minutes to anesthetize the animal before dissection begins.

2. Hold the anesthetized crayfish from behind the claws with one hand. Quickly, cut from the eye socket to the middle of the head on both sides, and then behead the crayfish (Note: the blood from the preparation will be sticky when it dries, so wash the tools when completed).

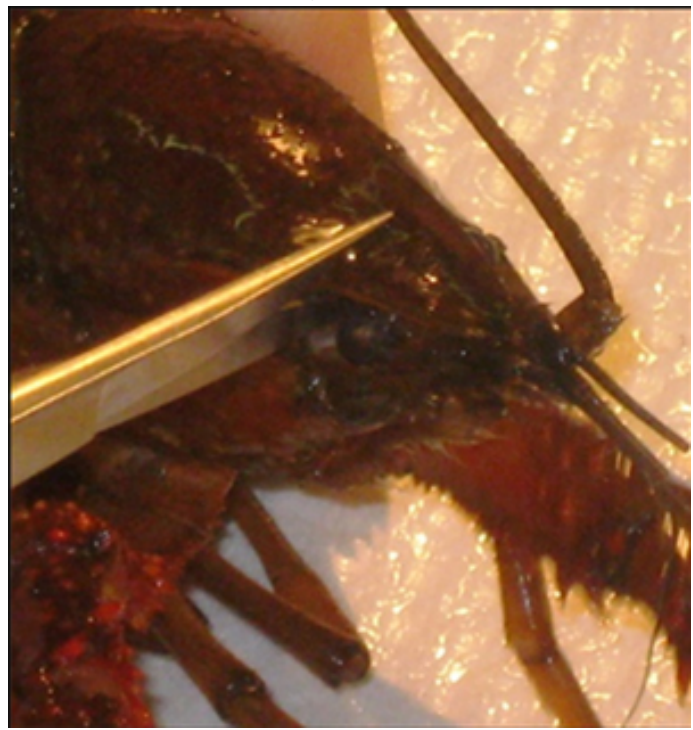

Figure 1: Decapitation of crayfish

3. Cut off the chelipeds and walking legs.

4. Cut off the left and right tailfins, only leaving the middle tailfin (uropod).

5. On the dorsal side of the crayfish cut ventrally on both the left and right side of the dorsal cuticle. 


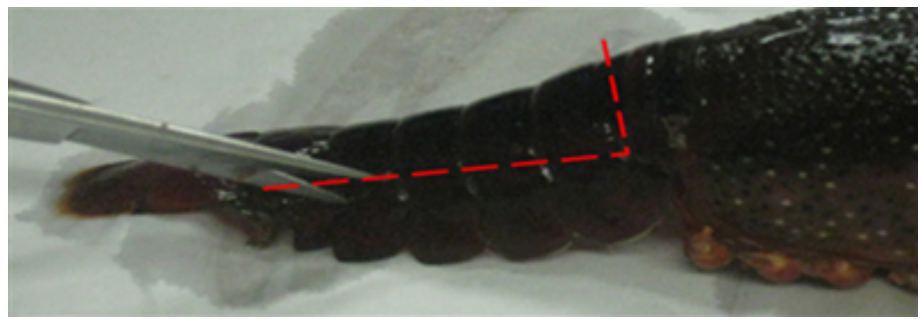

Figure 2: Removal of Dorsal Cuticle

6. Cut transversely on the dorsal side of the cuticle, making sure that the cut is shallow to prevent damage to the G.I. Then remove the lower section of the dorsal abdominal cuticle.

7. Place the dissected crayfish into a Sylgard-lined dish.

8. Pin the crayfish to the dish at the tip of the tailfin. One may use more pins on either side of the G.I. as necessary to hold the body down.

9. Fill the dish with crayfish saline covering the G.I. Make sure to continually douse the G.I. with saline using a pipette. Saline is a modified Van Harreveld's solution (1936), which is made with $205 \mathrm{mM} \mathrm{NaCl} ; 5.3 \mathrm{mM} \mathrm{KCl} ; 13.5 \mathrm{mM} \mathrm{CaCl} 2 \mathrm{H}_{2} \mathrm{O} ; 2.45 \mathrm{mM} \mathrm{MgCl}_{2} 6 \mathrm{H}_{2} \mathrm{O} ; 5 \mathrm{mM} \mathrm{HEPES}$ and adjusted to $\mathrm{pH}$ 7.4. The dissected crayfish should appear as shown in Figure 3.

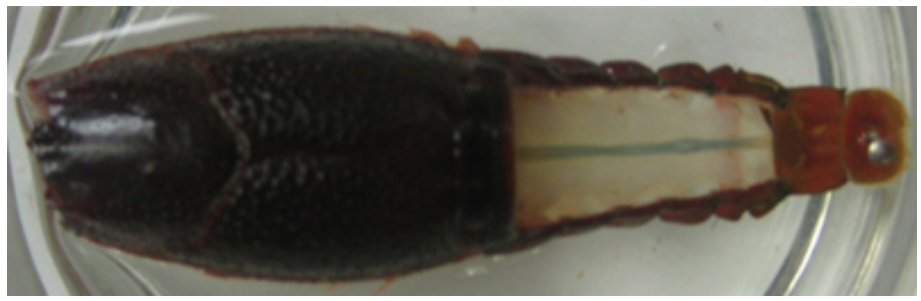

Figure 3: Dissected crayfish with GI intact.

\section{3) Experiments}

\subsection{1) Contractions in the animal}

1. Have solutions of the compound to be tested ready and at the same temperature as the bathing saline. On might use individual solutions of serotonin (100 nM, 1microM), glutamate (1microM), and dopamine (1microM) made in crayfish saline as starting substances to be tested.

2. Allow the dissected crayfish to sit in the crayfish solution for about ten minutes to allow it to adjust to the shock of dissection and saline exposure. The slow peristalsis contractions from the hindgut should start to occur.

3. Once the contractions begin, record the number of contractions that occur in thirty seconds (note the type of contractions that occur: i.e. peristalsis type, or spastic, and the direction of any peristaltic waves).

Table 1: Contractions in Crayfish Saline

\begin{tabular}{|l|l|}
\hline Number of Contractions & Type of Contractions \\
\hline & \\
\hline
\end{tabular}

4. Using a pipette, remove the saline within the cavity of the crayfish's exposed abdomen, and apply saline containing the substance to be examined directly onto the hindgut.

5. Immediately after adding the solution, record the number of contractions that occur after thirty seconds and note the type of contractions that occur (i.e. peristaltic, or spastic).

Table 2: Contractions with exposure to compounds

\begin{tabular}{|l|l|l|l|}
\hline Compound tested & Concentration & Number of Contractions & Type of Contractions \\
\hline & & & \\
\hline & & & \\
\hline & & & \\
\hline
\end{tabular}

6. Immediately after recording the response to the test substance, rinse the G.I. several times with normal crayfish saline. Let the preparation stand for 5 minutes, while rinsing about every 30 seconds with crayfish saline.

7. Using a pipette, place some saline containing the next compound to be examined or a varied concentration of the last substance tested directly onto the hindgut. It is best to start with a lower concentration and work one's way to higher concentrations.

8. Immediately after adding each new substance or each new concentration, record the number of contractions that occur after thirty seconds and note the type of contractions. 


\subsection{2) Recording forces of contraction in excised preparations}

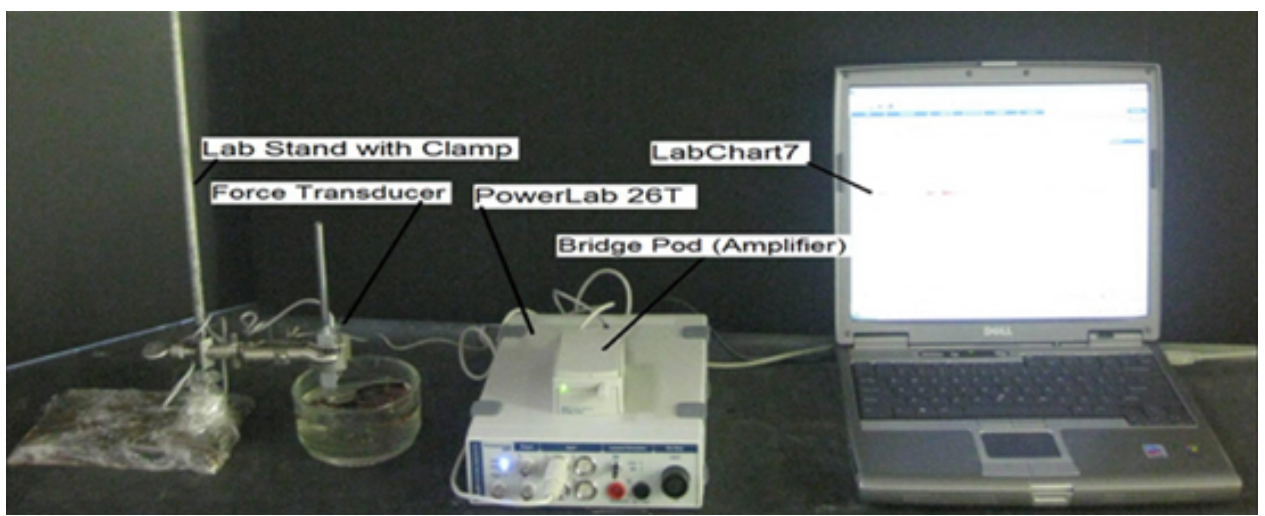

Figure 4: Setup

1. Attach the transducer to the bridge pod.

2. Attach the bridge pod to the PowerLab 26T.

3. Attach the PowerLab 26T to the USB port on the computer.

4. Open LabChart7, by clicking on the labchart7 icon on the desktop.

- The LabChart Welcome Center box will pop open. Close it.

- Click on Setup

- Click on channel settings. Change the number of channels to 1 (bottom left of box) push OK.

- At the top left of the chart set the cycles per second to about $2 \mathrm{k}$. Set the volts (y-axis) to about 500 or $200 \mathrm{mV}$.

- Click on Channel 1 on the right of the chart. Click on Input Amplifier. Ensure that the settings: single-ended, ac coupled, and invert (inverts the signal if needed), and anti-alias, are checked.

- To begin recording press start.

5. Pick up the preparation and cut the GI tract at the juncture between the thorax and the abdomen. Then carefully cut around the telson part of the tail. Remove the GI tract from the dissected crayfish and place it into the Sylgard dish. There is a blood vessel that runs along the dorsal aspect of the GI tract. Carefully pull this away from the GI tract. Pour fresh crayfish saline onto the preparation.

6. Place the force transducer in the clamp near the dissected crayfish.

7. Hook the force transducer in the crayfish G.I. as shown in Figure 5.

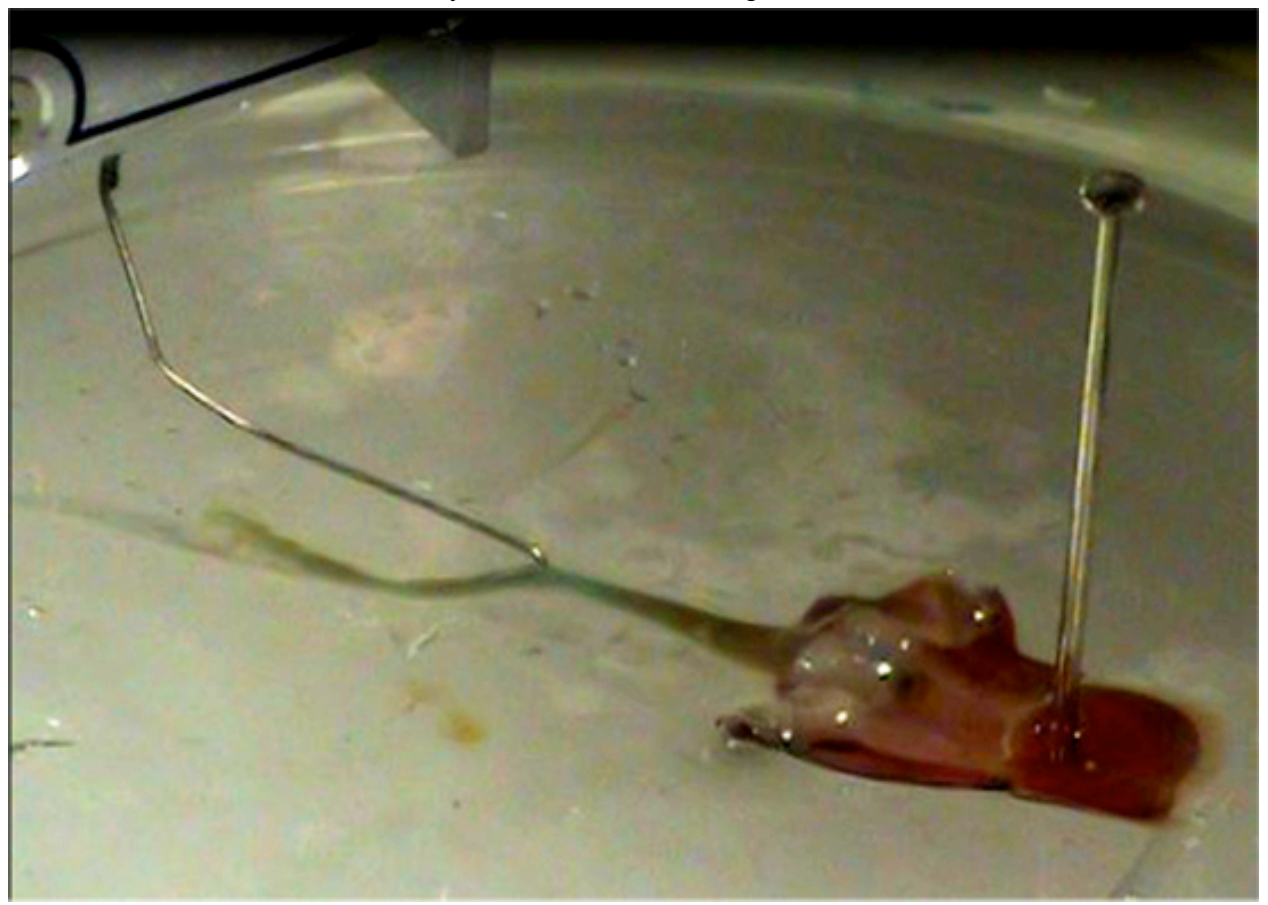

Figure 5: Isolated hindgut of the crayfish in saline attached to the hook of the force transducer

8. Wait until the G.I. starts to contract, and push start on the LabChart7.

9. Let the program run for about 20 seconds. Push "stop," and add a comment labeled, "Saline only". Fill out the Table 4.

Table 4: Contractions with compound of interest

\begin{tabular}{|l|l|}
\hline Number of Contractions & Amplitude of Contractions $(\mathrm{mV})$ \\
\hline
\end{tabular}


0. Add the test compounds of interest, and immediately push "start" on the LabChart7.

11. Let the program run for about 20 seconds. Push "stop" and add a comment directly on the chart file as to what substance was added and its concentration. Fill out the table below.

Table 5: Contractions with compound

\begin{tabular}{|l|l|}
\hline Number of Contractions & Amplitude of Contractions (mV) \\
\hline & \\
\hline
\end{tabular}

12. Rinse off the preparation with crayfish saline using a pipette.

13. Add other substances or various concentrations in a similar manner. Immediately push "start" on the LabChart7.

14. Let the program run for about 20 seconds. Push "stop," and add a comment and label directly on the chart file indicating the compound used and the concentration.

\section{Data Analysis}

1. From 2.3.1 of the experiment, graph the number of contractions from each solution (saline, serotonin, glutamate) vs. time. Explain any trends.

2. From 2.3.2 of the experiment, graph the number of contractions from each solution (saline, serotonin, glutamate) vs. the amplitude of the contractions in $\mathrm{mV}$. Explain any trends.

\section{1) Measuring the rate and force of contractions}

To index the rates of contractions on can measure the time from the beginning on one deflection to the beginning of the next or count the total deflections over a minute or two. The values can then be put in terms of contractions per minute or per second depending on how rapid they are occurring.

To index the force of contractions a relative measure could be used to compare different conditions. On the saved file one can use the marker "M" and move to the baseline. Then use the cursor and move to the peak of the deflection and take note of the value listed on the top right of the screen as a delta value (the difference from $\mathrm{M}$ to the peak). Note the cursor needs to be kept stationary for the measure of the change. Then move the $M$ to the next wave form of interest and repeat the measure.

\section{Discussion}

The details provided in the associated movie and text describe the key steps needed to record the activity in the hindgut of the crayfish in situ as well as in vitro. One goal of our report is to increase the awareness to the potential of this preparation in student-run investigative laboratories that teach fundamental concepts in physiology and pharmacology.

These preparations can be used to investigate a number of experimental questions that will lead to a better understanding of the physiological functions of the hindgut. The mechanisms underlying regulation of peristaltic waves and their reversal are still not known. The mechanisms for higher control of the entire GI tract are also not fully understood. The question of how higher centers integrate their activity with the autonomic output that directly controls the GI system remains an open area of investigation (Shuranova et al., 2006). In addition, the osmoregulatory capabilities of the crustacean hindgut and the functions of osmoregulation during molting and environmental stress have not been fully elucidated. There are still many questions awaiting answers in this preparation.

\section{Disclosures}

No conflicts of interest declared.

\section{Acknowledgements}

Supported by University of Kentucky, Department of Biology, Office of Undergraduate Studies and College of Arts \& Sciences.

\section{References}

1. Alexandrowicz, J. S. Zur Kenntnis des sympathischen Nervensystems der Crustaceae. Jena Z. Naturw. 45, 395-444 (1909).

2. Bungart, D., Dircksen, H. \& Keller, R. Quantitative determination and distribution of the myotropic neuropeptide orcokinin in the nervous system of astacidean crustaceans. Peptides 15, 393-400 (1994).

3. Dircksen, H., Burdzik, S., Sauter, A. \& Keller, R. Two orcokinins and the novel octapeptide orcomyotropin in the hindgut of the crayfish Orconectes limosus: identified myostimulatory neuropeptides originating totether in neurons of the terminal abdominal ganglion. J. Exp. Biol. 203, 2807-2818 (2000).

4. Elekes, K., Florey, E., Cahil, M.A., Hoeger, U. \& Geffard, M. Morphology, synaptic connections and neurotransmitters of the efferent neurons of the crayfish hindgut. In: Salanki J, Rosza K (eds) Neurobiology of Invertebrates, Vol. 36, Akademiai Kiado, Budapest, pp 129-146 (1988).

5. Elofsson, R., Kauri, T., Nielsen, S.O. \& Stroemberg, J.O. Catecholamine-containing nerve fibres in the hindgut of the crayfish Astacus astacus L. Experentia 24, 1159-1160 (1968).

6. Florey, E. Uber die wirkung von acetylcholin, adrenalin, nor-adrenalin, faktor I und anderen substanzen auf den isolierten enddarm des flusskrebses Cambarus clarkii Girard. Z. Vergl. Physiol. 36, 1-8 (1954). 
7. Florey, E. A new test preparation for bio-assay of Factor I and gamma-aminobutyric acid. J. Physiol. 156, 1-7 (1961).

8. Jones, H.C. The action of L-glutamic acid and of structurally related compounds on the hind gut of the crayfish. J. Physiol. (London) 164 , 295-300 (1962).

9. Knotz, S. \& Mercier, A.J. cAMP mediates dopamine-evoked hindgut contractions in the crayfish, Procambarus clarkii. Comp. Biochem. Physiol.111A, 59-64 (1995).

10. Kondoh, Y. \& Hisada, M. Neuroanatomy of the terminal (sixth abdominal) ganglion of the crayfish, Procambarus clarkii (Girard). Cell. Tiss. Res. 243, 273-288 (1986).

11. Mercier, A.J., Lange, A.B., TeBrugge, V. \& Orchard, I. Evidence for proctolin-like and FMRFamide-like neuropeptides associated with the hindgut of the crayfish, Procambarus clarkii Can. J. Zool. 75, 1208-1225 (1997).

12. Mercier, A.J. \& Lee, J. Differential effects of neuropeptides on circular and longitudinal muscles of the crayfish hindgut. Peptides 23 , 1751-1757 (2002).

13. Mercier, A.J., Orchard, I. \& Schmoeckel, A. Catecholaminergic neurons supplying the hindgut of the crayfish, Procambarus clarkii. Can. J. Zool. 69, 2778-2785 (1991a).

14. Mercier, A.J., Orchard, I. \& TeBrugge, V. FMRFamide-like immunoreactivity in the crayfish nervous system. J. exp. Biol. 156, 519-538 (1991b).

15. Mercier, A.J., Orchard, I., TeBrugge, V \& Skerrett, M. Isolation of two FMRFamide-related peptides from crayfish pericardial organs. Peptides $14,137-143(1993)$.

16. Musolf, B.E. Serotonergic modulation of the crayfish hindgut: Effects on hindgut contractility and regulation of serotonin on hindgut. Dissertation (2007). (Downloaded from an open access source at Georgia State University; http://etd.gsu.edu/theses/available/ etd-11272007-175638/ )

17. Musolf, B.E., Spitzer, N., Antonsen, B.L. \& Edwards, D.H. Serotonergic modulation of crayfish hindgut. Biol Bull. 217(1), 50-64 (2009).

18. Shuranova, Z.P., Burmistrov, Y.M., \& Cooper, R.L. A hundred years ago and now: A short essay on the study of the crustacean hindgut. (Vor hundert Jahren und nun: Eine kurze Geschichte von die Forschung des Hinterdarmes der Crustaceen). Crustaceana 76, 755-670 (2003).

19. Shuranova, Z.P., Burmistrov, Y.M., Strawn, J.R. \& Cooper, R.L. Evidence for an Autonomic Nervous System in Decapod Crustaceans. International Journal of Zoological Research 2(3), 242-283 (2006).

20. Siwicki, K.K. \& Bishop, C.A. Mapping of proctolinlike immunoreactivity in the nervsou systems of lobster and crayfish. J. comp. Neurol. 243 , 435-453 (1986).

21. Stemmler, E.A., Cashman, C.R., Messinger, D.I., Gardner, N.P., Dickinson, P.S. \& Christie, A.D. High-mass-resolution direct-tissue MALDIFTMS reveals broad conservation of three neuropeptides (APSGFLGMRamide, GYRKPPFNGSIFamide and pQDLDHVFLRFamide) across members of seven decapod crustaean infraorders. Peptides 28, 2104-2115 (2007).

22. Wales, W. Control of mouthparts and gut. In: The Biology of Crustacea, Vol. 4; D.C. Sandeman and H.L. Atwood, eds.; Academic Press Inc., London; pp. 165-191 (1982).

23. Webster, S.G., Dircksen, H. \& Chung, J.S. Endocrine cells in the gut of the shore crab Carcinus maenas immunoreactive to crustacean hyperglycaemic hormone and its precursor-related peptide. J. Exp. Biol. 300, 193-205 (2000).

24. Winlow, W. \& Laverack, M.S. The control of hindgut motility in the lobster Homarus gammarus (L.). 1. Analysis of hindgut movements and receptor activity. Mar. Behav. Physiol. 1, 1-28 (1972a).

25. Winlow, W. \& Laverack, M.S. The control of hindgut motility in the lobster Homarus gammarus (L.). 2. Motor output. Mar. Behav. Physiol. 1, $29-47(1972 b)$

26. Winlow, W. \& Laverack, M.S. The control of hindgut motility in the lobster Homarus gammarus (L.). 3. Structure of the sixth abdominal ganglion (6 A.G.) and associated ablation and microelectrode studies. Mar. Behav. Physiol. 1, 93-121 (1972c).

27. Wrong, A.D., Sammahin, M., Richardson, R. \& Mercier, A.J. Pharmacological properties of glutamate receptors associated with the crayfish hindgut. J. Comp. Physiol. 189, 371-378 (2003). 